\title{
Reaction Outcome Critically Dependent on the Method of Workup: An Example from the Synthesis of 1-Isoquinolones
}

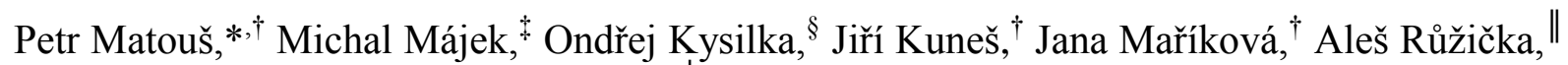
Milan Pour, ${ }^{, \dagger}$ and Pavel Kočovský ${ }^{* \dagger},, \nabla, \perp$

†Department of Bioorganic and Organic Chemistry, Faculty of Pharmacy in Hradec Králové, Charles University, Heyrovského 1203, 50005 Hradec Králové, Czech Republic

Department of Organic Chemistry, Faculty of Natural Sciences, Comenius University, Mlynská dolina, 84215 Bratislava 4, Slovakia

${ }^{\S}$ Trelleborg Bohemia, Akademika Bedrny 531/8a, Věkoše, 50003 Hradec Králové, Czech Republic

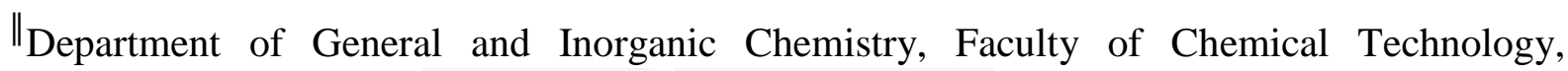
University of Pardubice, Studentská 95, 53210 Pardubice 2, Czech Republic

จ Department of Organic Chemistry, Faculty of Science, Charles University, Hlavova 8, 12843 Prague 2, Czech Republic

${ }^{\perp}$ Institute of Organic Chemistry and Biochemistry, Academy of Sciences of the Czech Republic, Flemingovo 2, 16610 Prague 6, Czech Republic

P. Matouš: matousp1@faf.cuni.cz

M. Májek:michal.majek@uniba.sk

O. Kysilka: ondrej.kysilka@yandex.com

J. Kuneš: kunes@ faf.cuni.cz

J. Maříková: marikoj2@faf.cuni.cz

A. Růžička: ales.ruzicka@upce.cz

M. Pour: pour@faf.cuni.cz

P. Kočovský: pavel.kocovsky@natur.cuni.cz

\section{Table of Contents}

${ }^{1} \mathrm{H}$, and ${ }^{13} \mathrm{C}\left\{{ }^{1} \mathrm{H}\right\}$ Spectra of New Compounds 


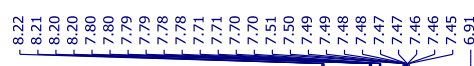

${ }^{1} \mathrm{H}$ NMR

$500 \mathrm{MHz}$,

DMSO-d $_{6}$

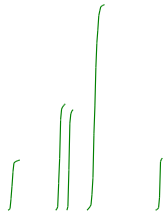<smiles>O=c1[nH]c(-c2ccccc2)cc2ccccc12</smiles>

$2 a$

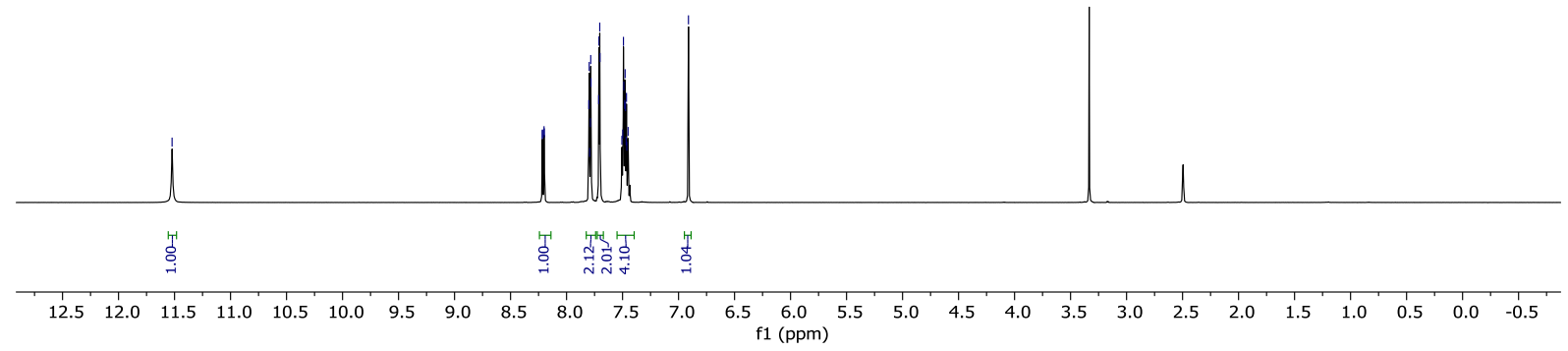

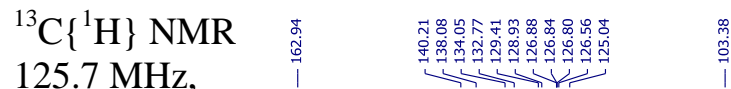

DMSO-d $_{6}$<smiles>O=c1[nH]c(-c2ccccc2)cc2ccccc12</smiles>

$2 a$

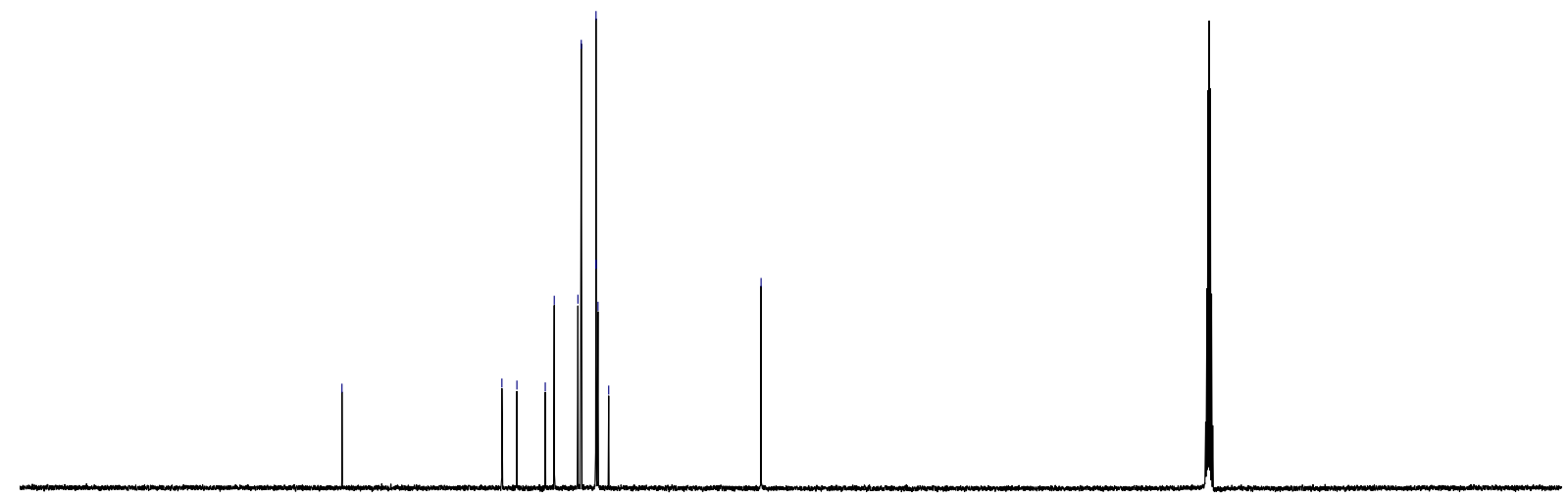

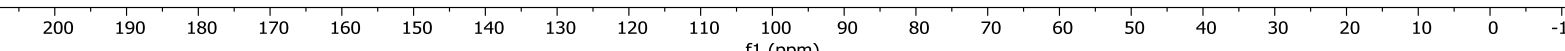




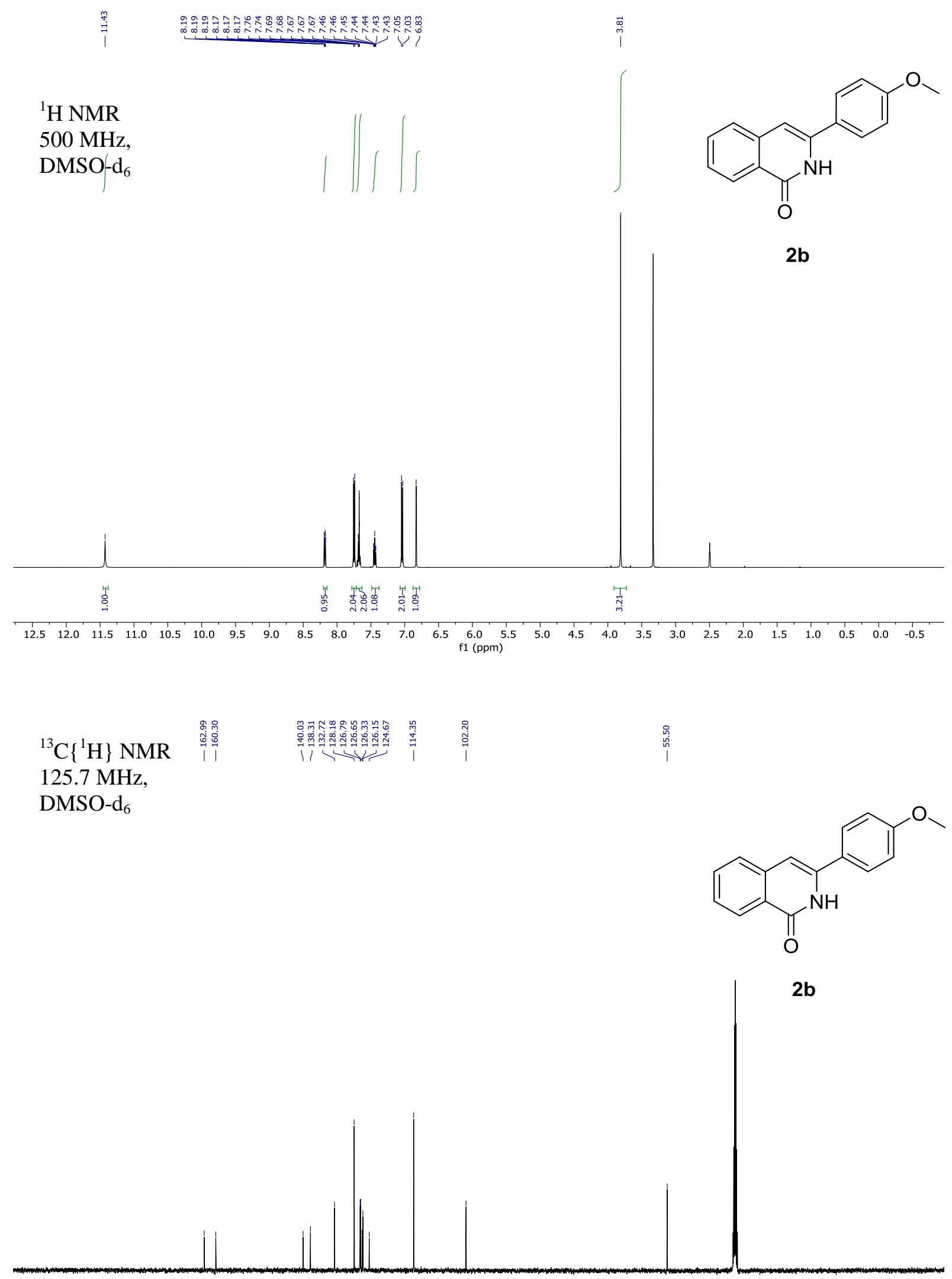

${ }^{13} \mathrm{C}\left\{{ }^{1} \mathrm{H}\right\}$
125.7
DMSO-d

$500 \mathrm{MHz}$,

D

2b 


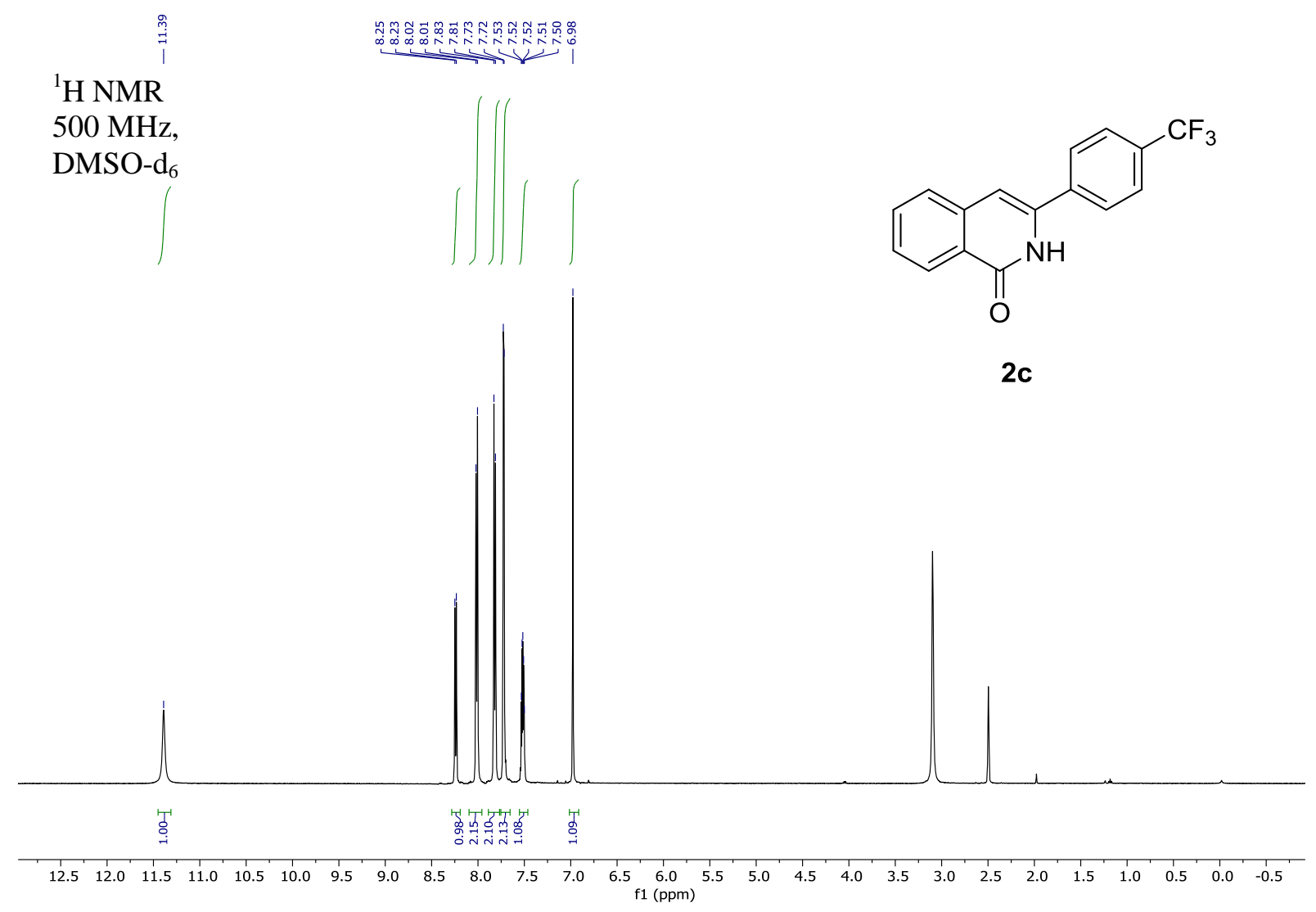

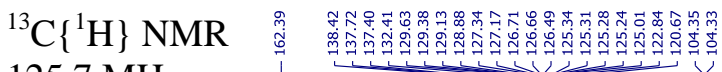

125.7 MHz,

$\mathrm{DMSO}_{6}$

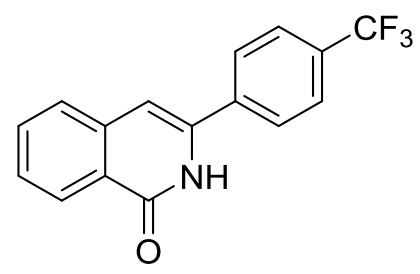

2c

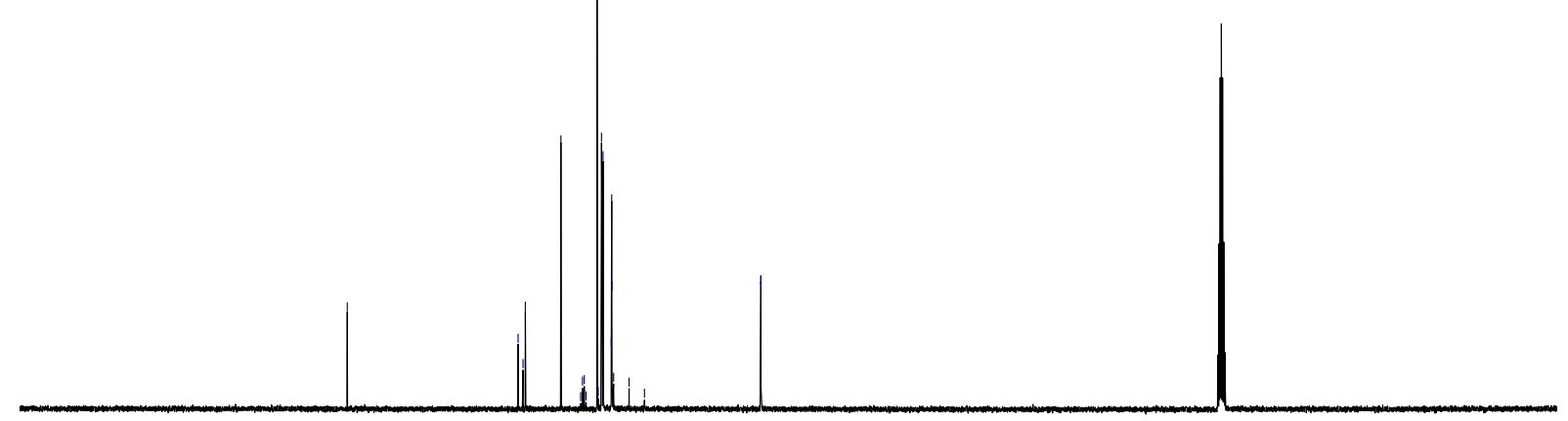

$\begin{array}{lllllllllll}200 & 190 & 180 & 170 & 160 & 150 & 140 & 130 & 120 & 110 & 100 \\ \mathrm{f} 1(\mathrm{ppm})\end{array}$ 


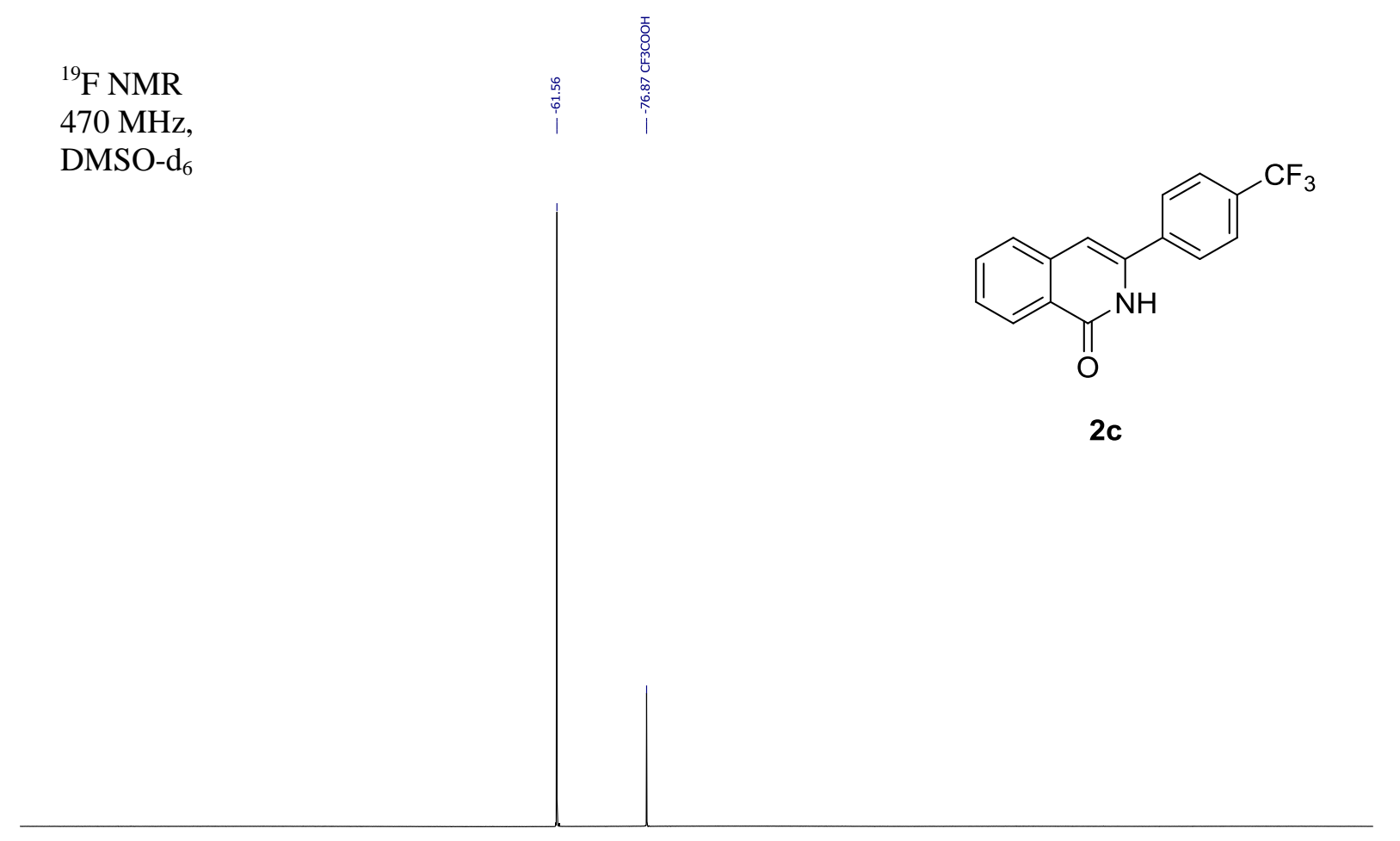

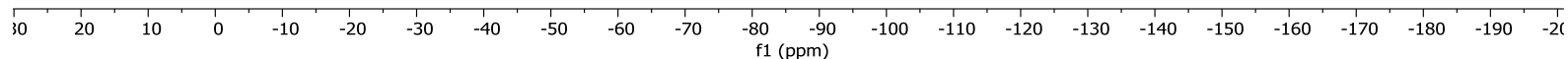


${ }^{1} \mathrm{H}$ NMR

$500 \mathrm{MHz}$,

DMSO-d $_{6}$
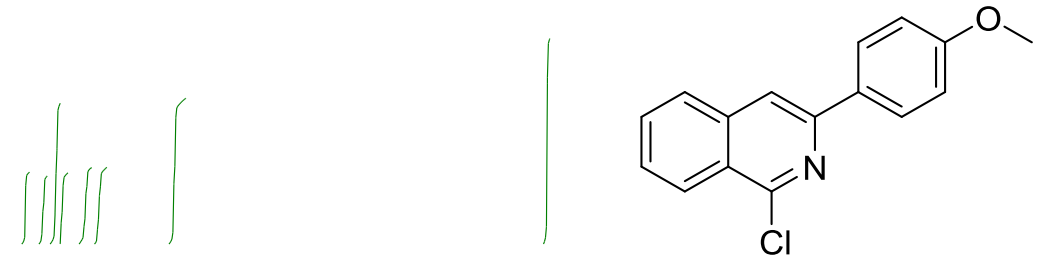

3b

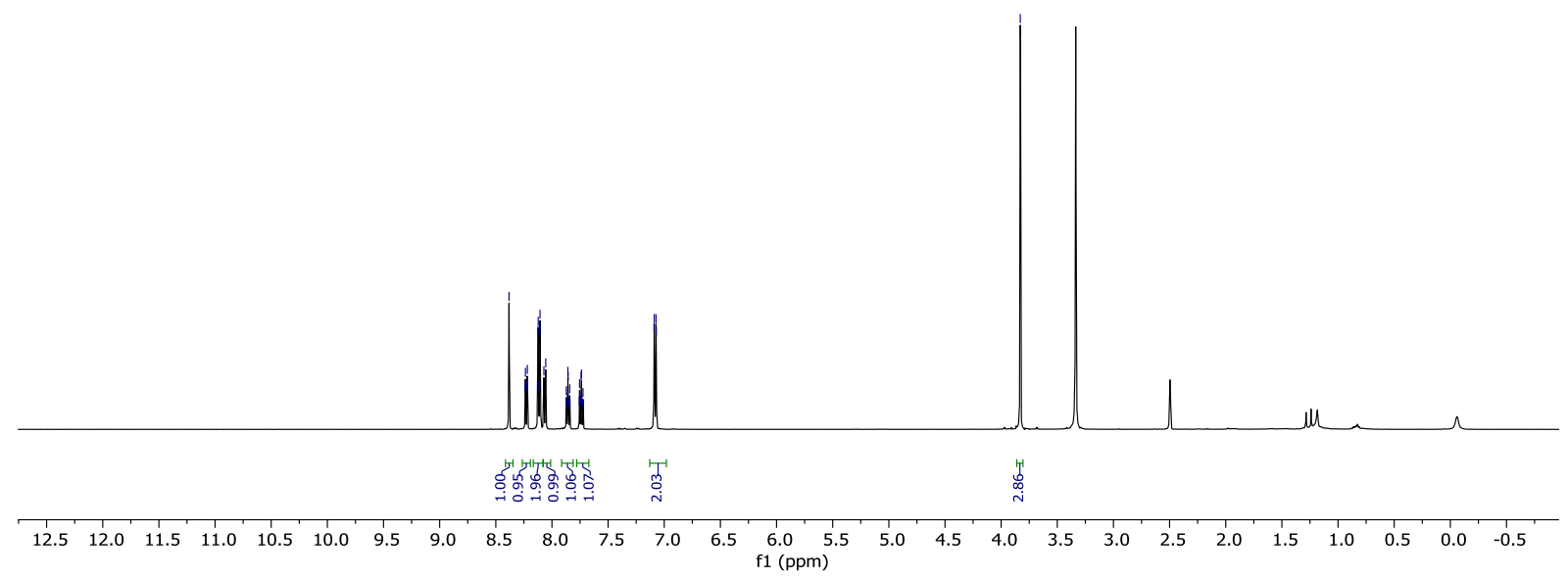

${ }^{13} \mathrm{C}\left\{{ }^{1} \mathrm{H}\right\} \mathrm{NMR}$ ।

125.7 MHz,

DMSO-d $_{6}$<smiles>COc1ccc(-c2cc3ccccc3c(Cl)n2)cc1</smiles>

$3 b$

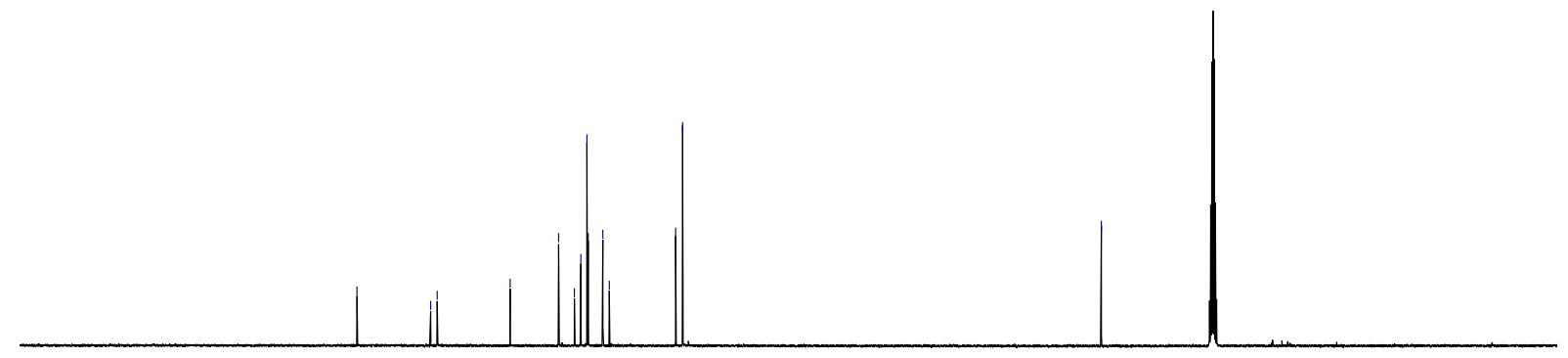

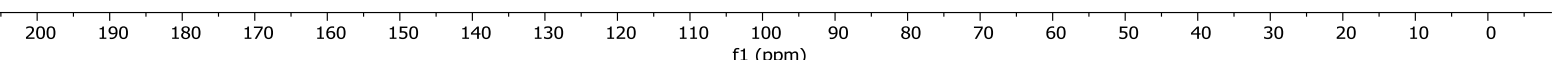




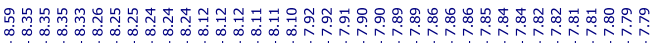

${ }^{1} \mathrm{H}$ NMR

$500 \mathrm{MHz}$,

DMSO-d $_{6}$
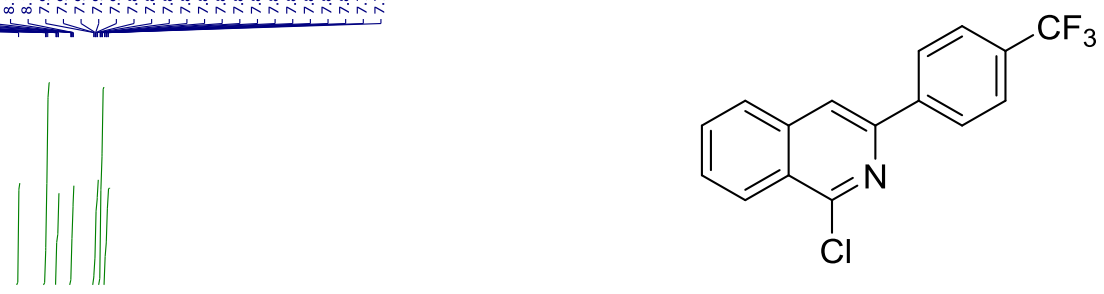

$3 c$

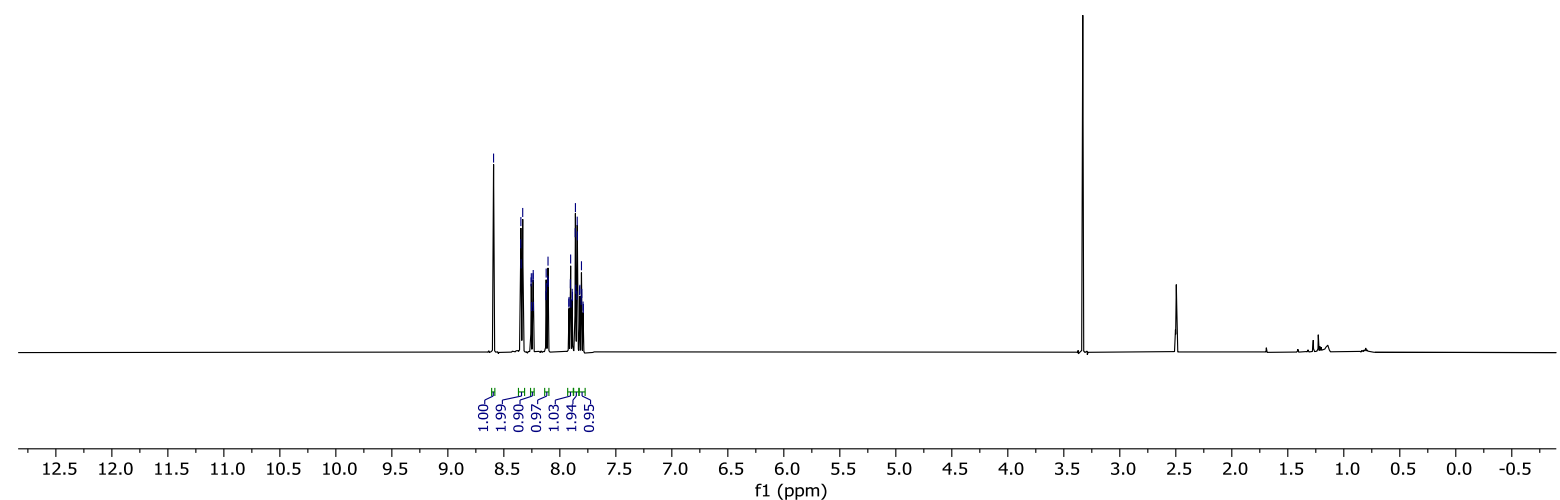

${ }^{13} \mathrm{C}\left\{{ }^{1} \mathrm{H}\right\} \mathrm{NMR} \quad{ }^{q}{ }^{m}$ o

125.7 MHz,

DMSO-d $_{6}$
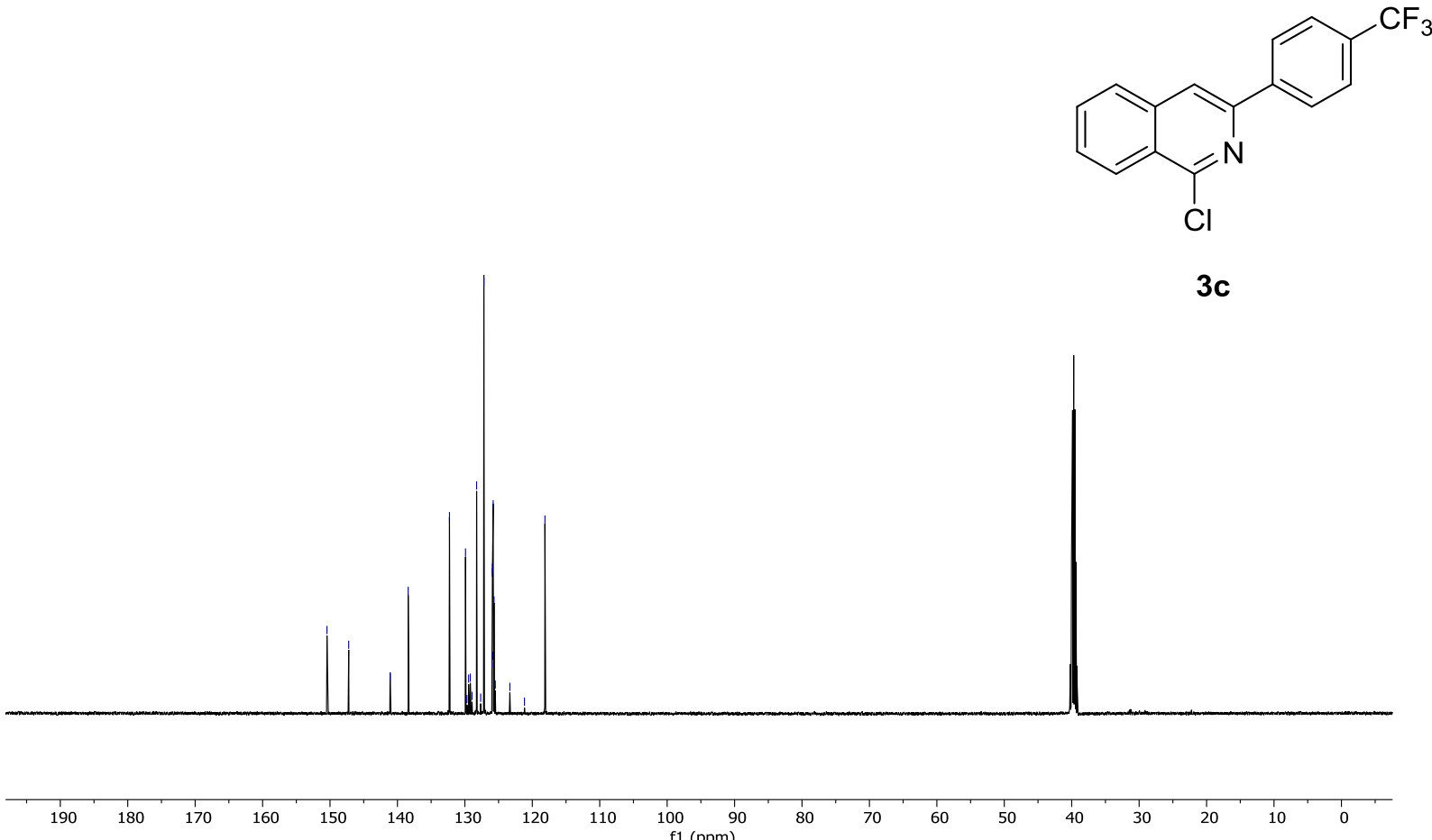
${ }^{19} \mathrm{~F}$ NMR

$470 \mathrm{MHz}$,

$\mathrm{DMSO}^{-\mathrm{d}_{6}}$
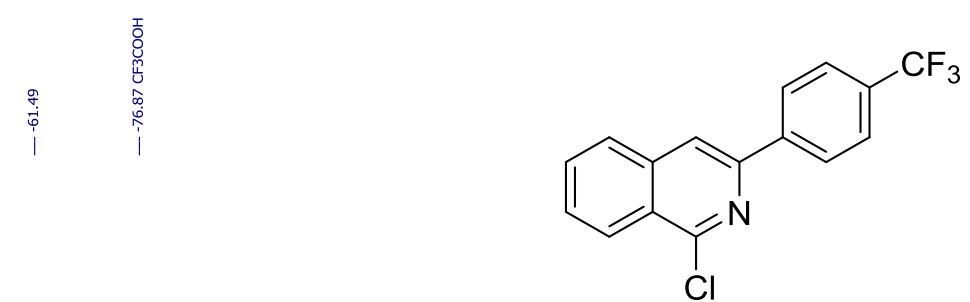

$3 c$

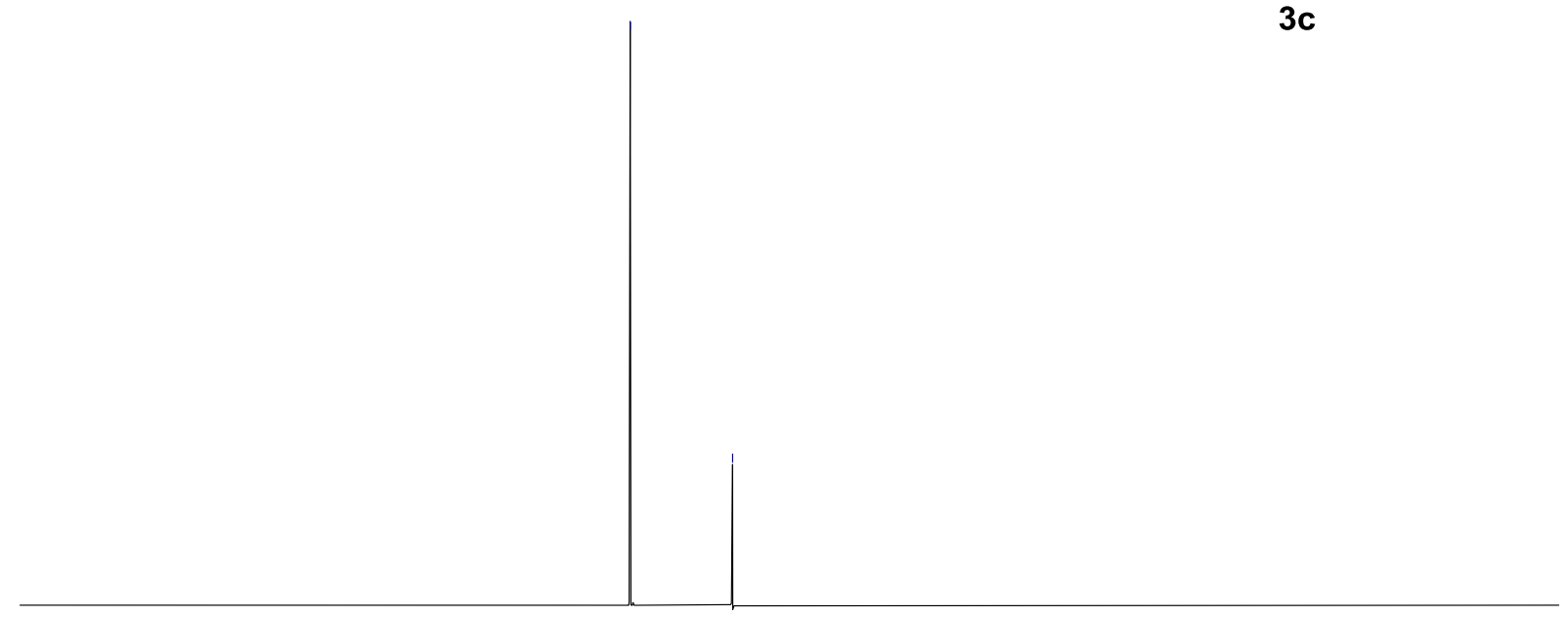

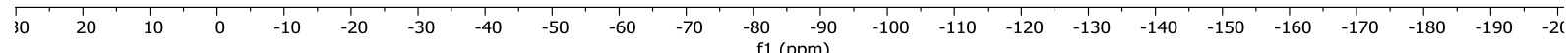



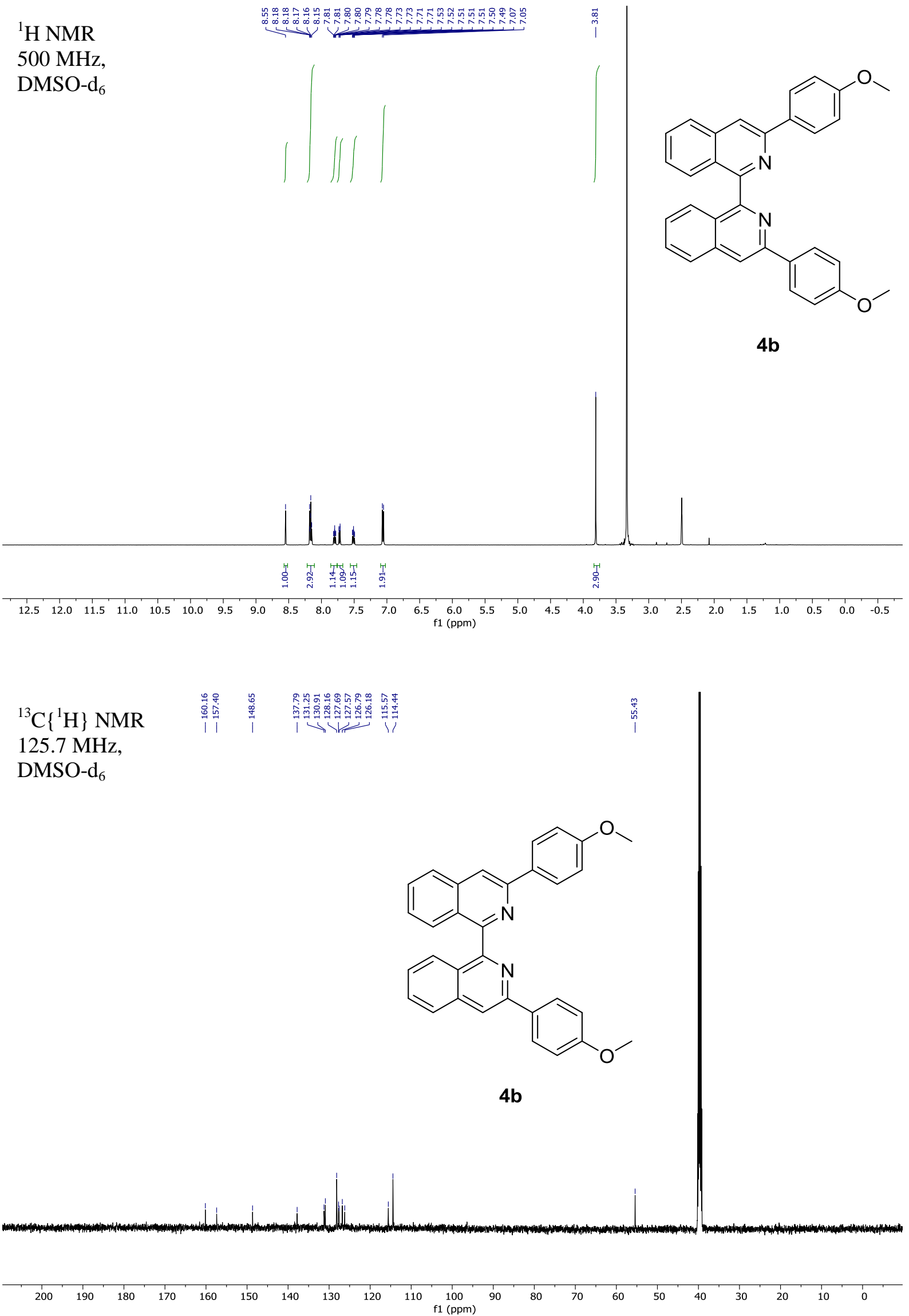
${ }^{1} \mathrm{H}$ NMR

$500 \mathrm{MHz}$

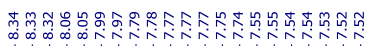

CDC1.
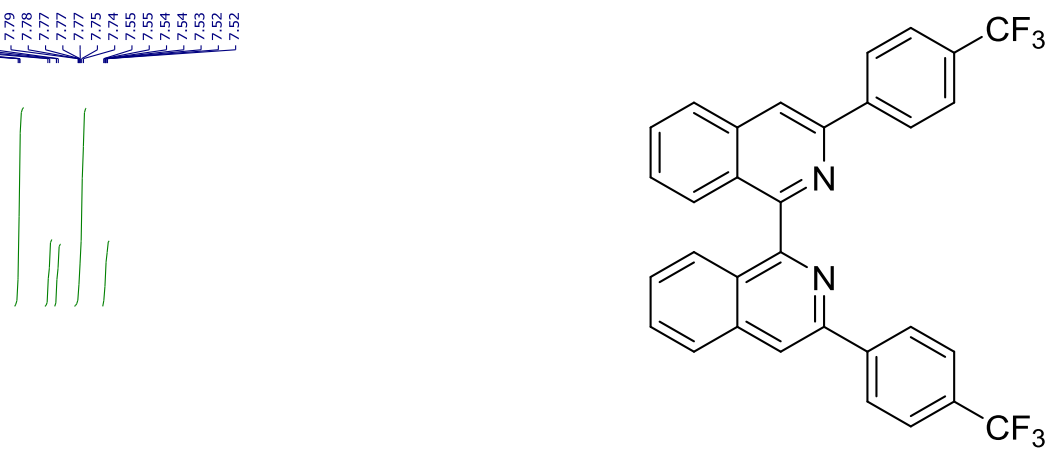

4c

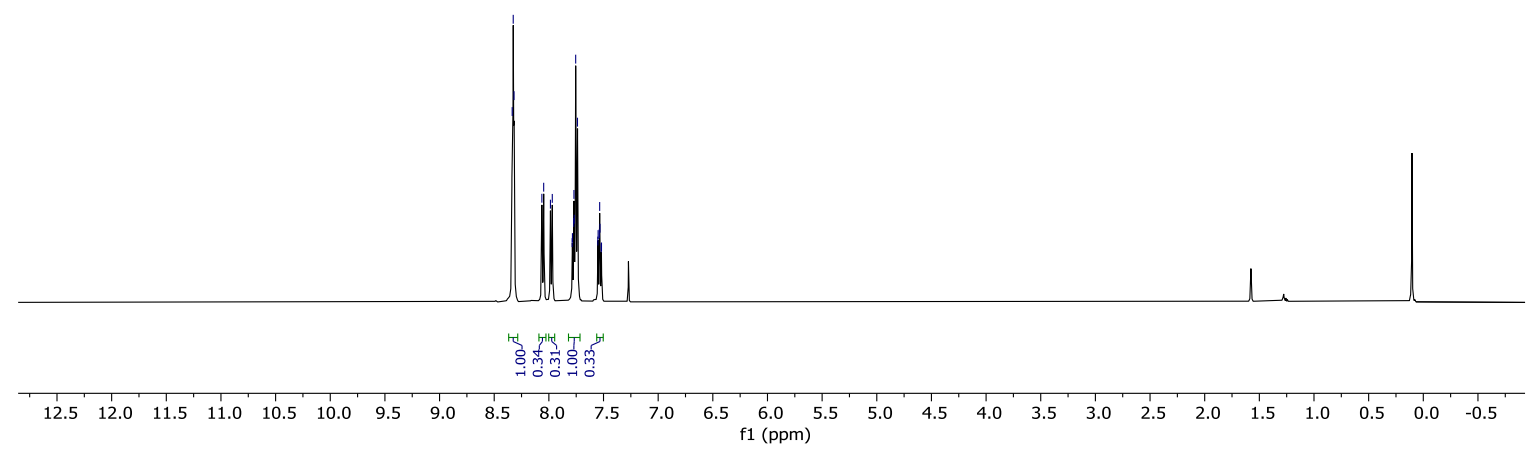

${ }^{13} \mathrm{C}\left\{{ }^{1} \mathrm{H}\right\} \mathrm{NMR}$

$125.7 \mathrm{MHz}$

$\mathrm{CDCl}_{2}$

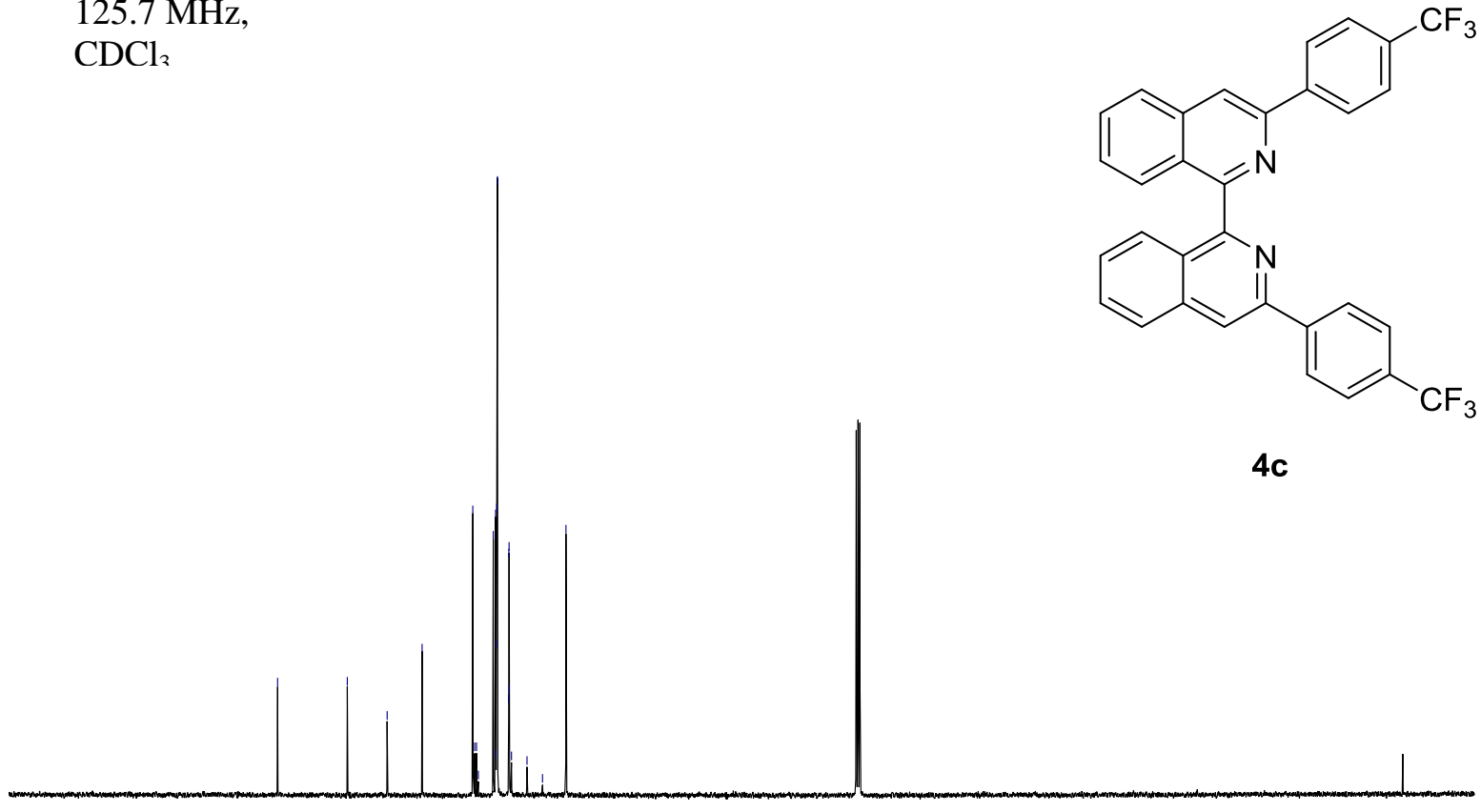

4c 


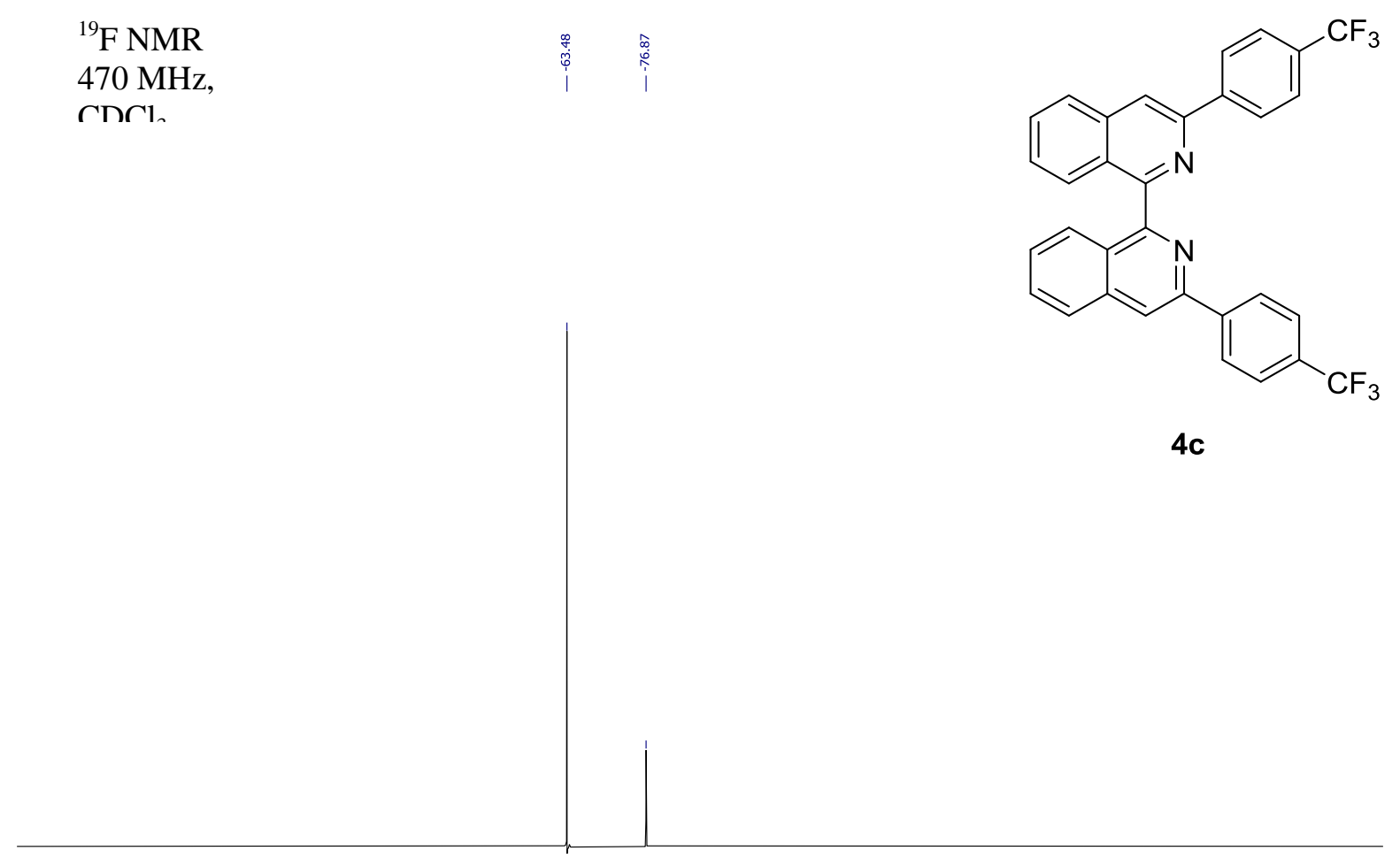

\begin{tabular}{llllllllllllllllllllllllllllll}
\hline & 1 & 10 & 0 & -10 & -20 & -30 & -40 & -50 & -60 & -70 & -80 & -90 & -100 & -110 & -120 & -130 & -140 & -150 & -160 & -170 & -180 & -190 & -20 \\
\hline
\end{tabular} 


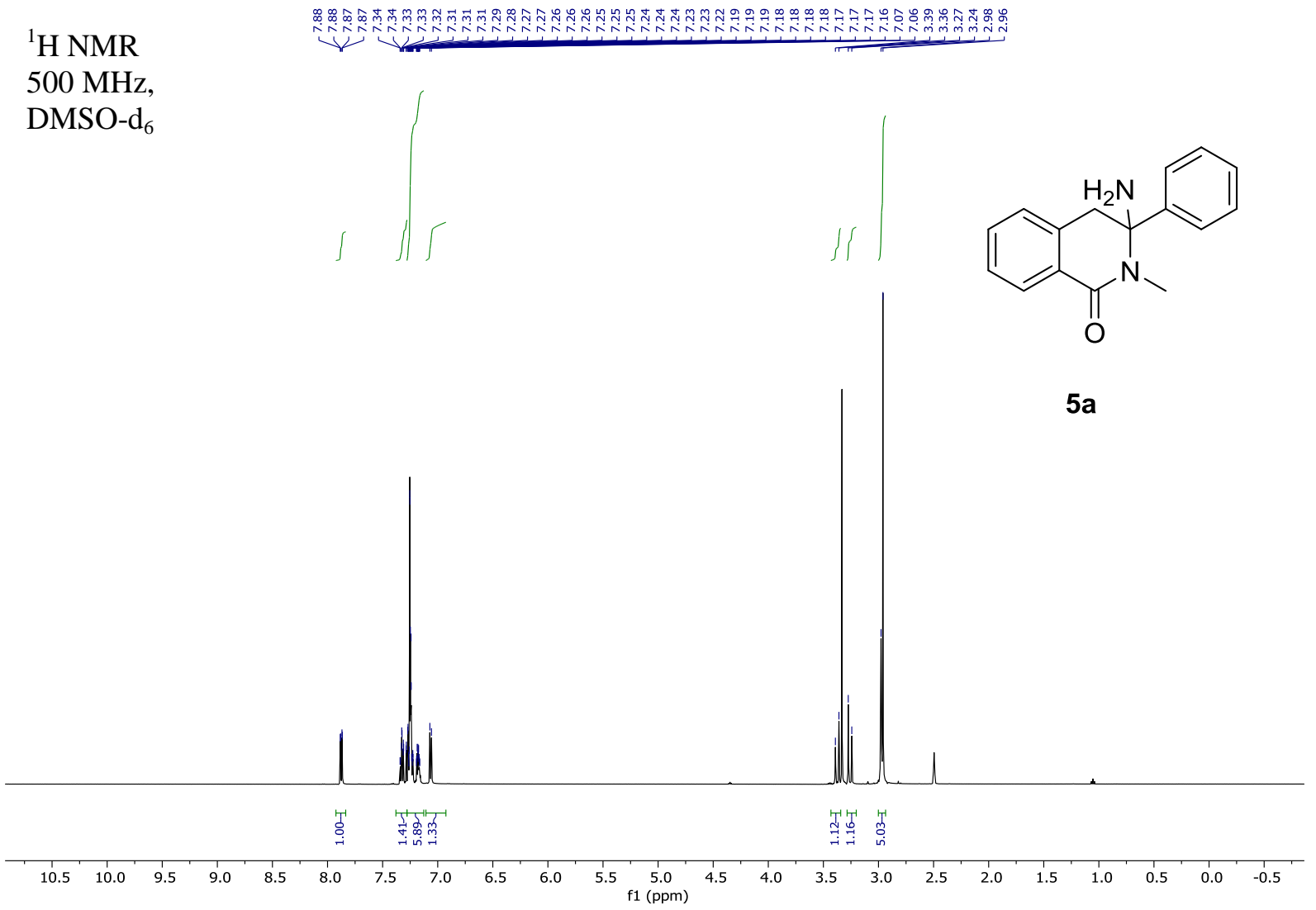

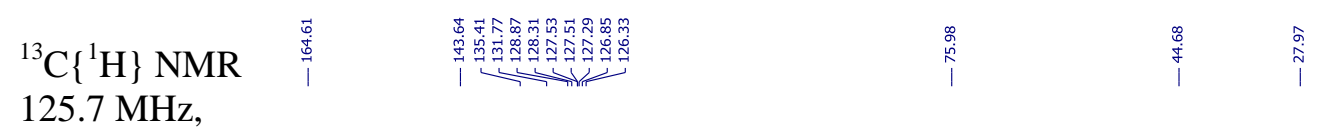

DMSO-d $_{6}$<smiles>CN1C(=O)c2ccccc2CC1(N)c1ccccc1</smiles>

$5 a$

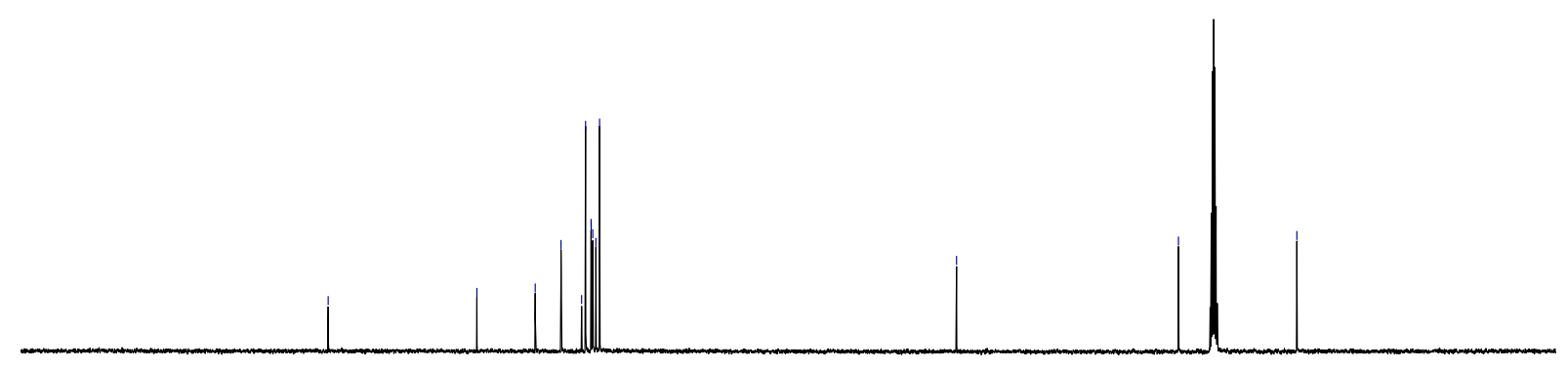

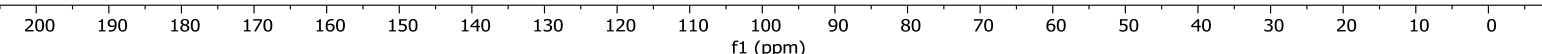


${ }^{1} \mathrm{H}$ NMR

$500 \mathrm{MHz}$, DMSO-d $_{6}$

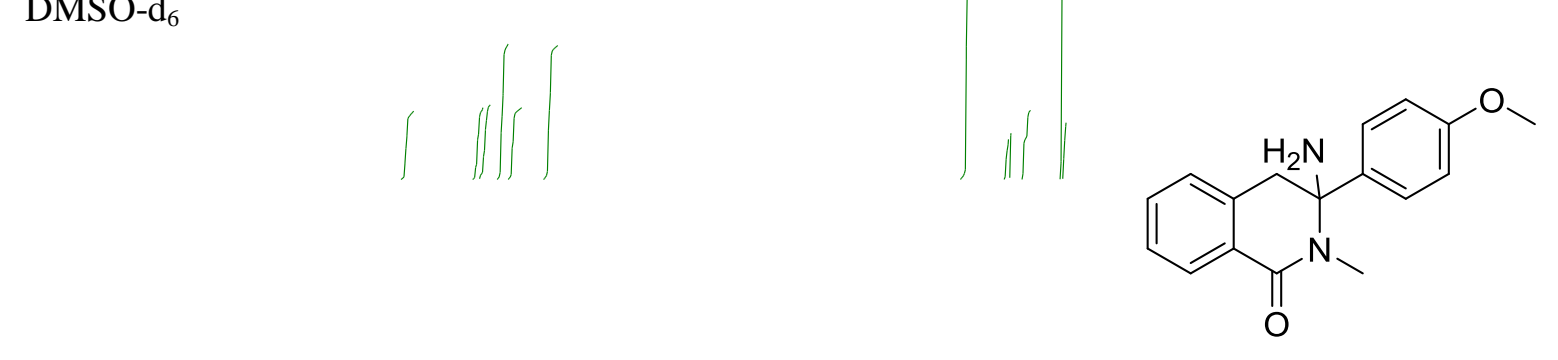

$5 b$

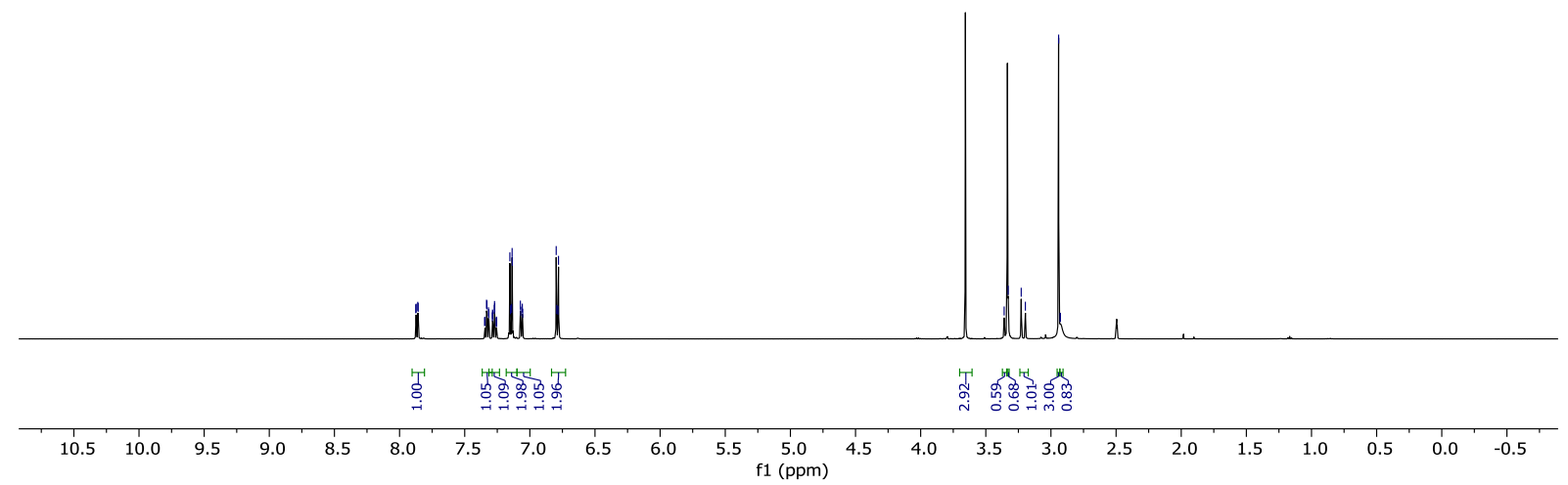

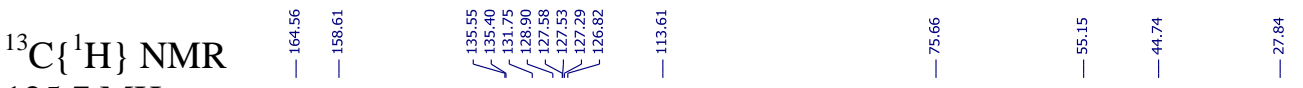

125.7 MHz,

DMSO-d $_{6}$

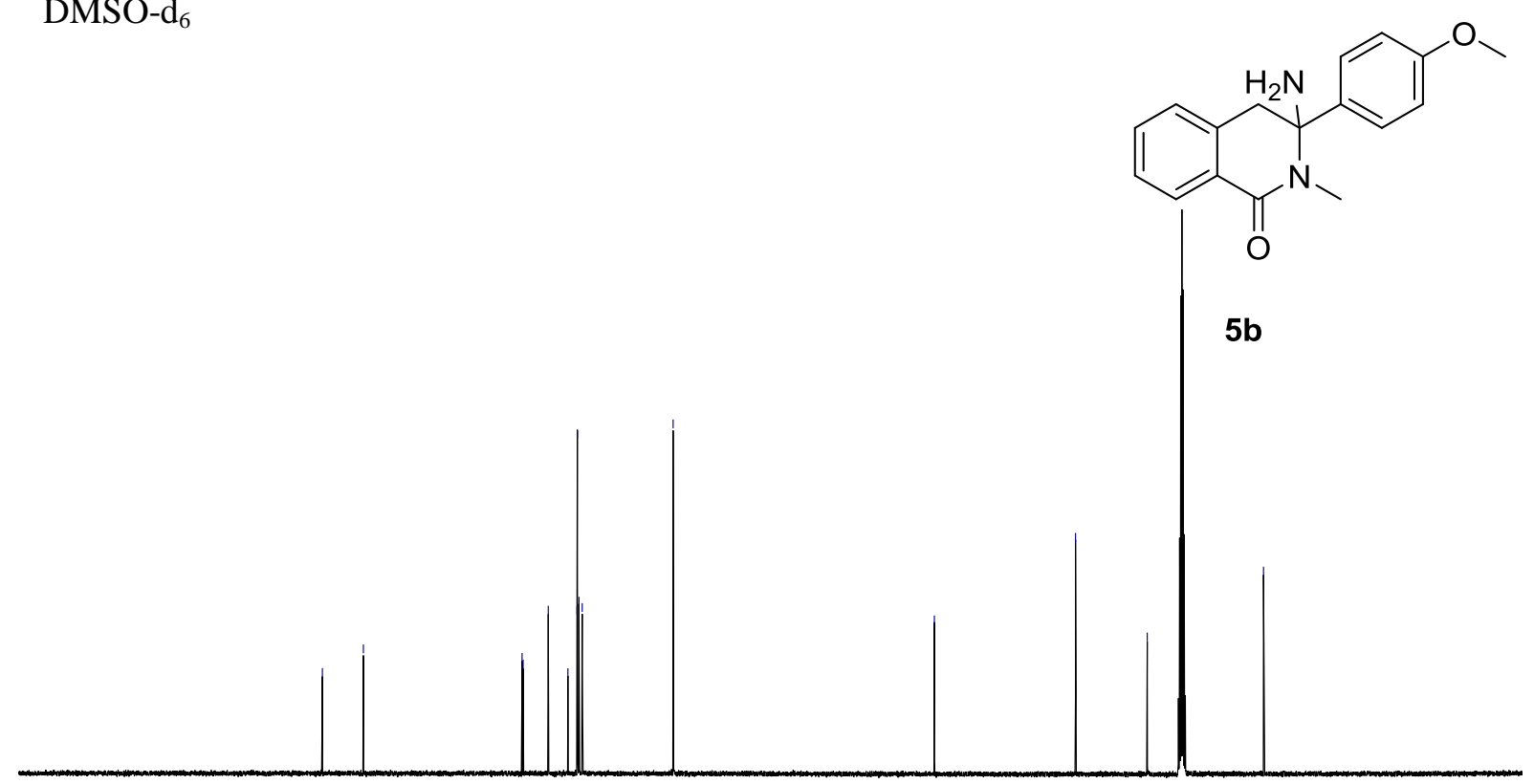

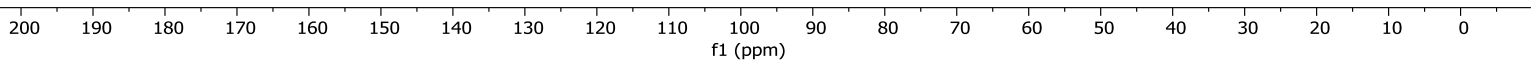




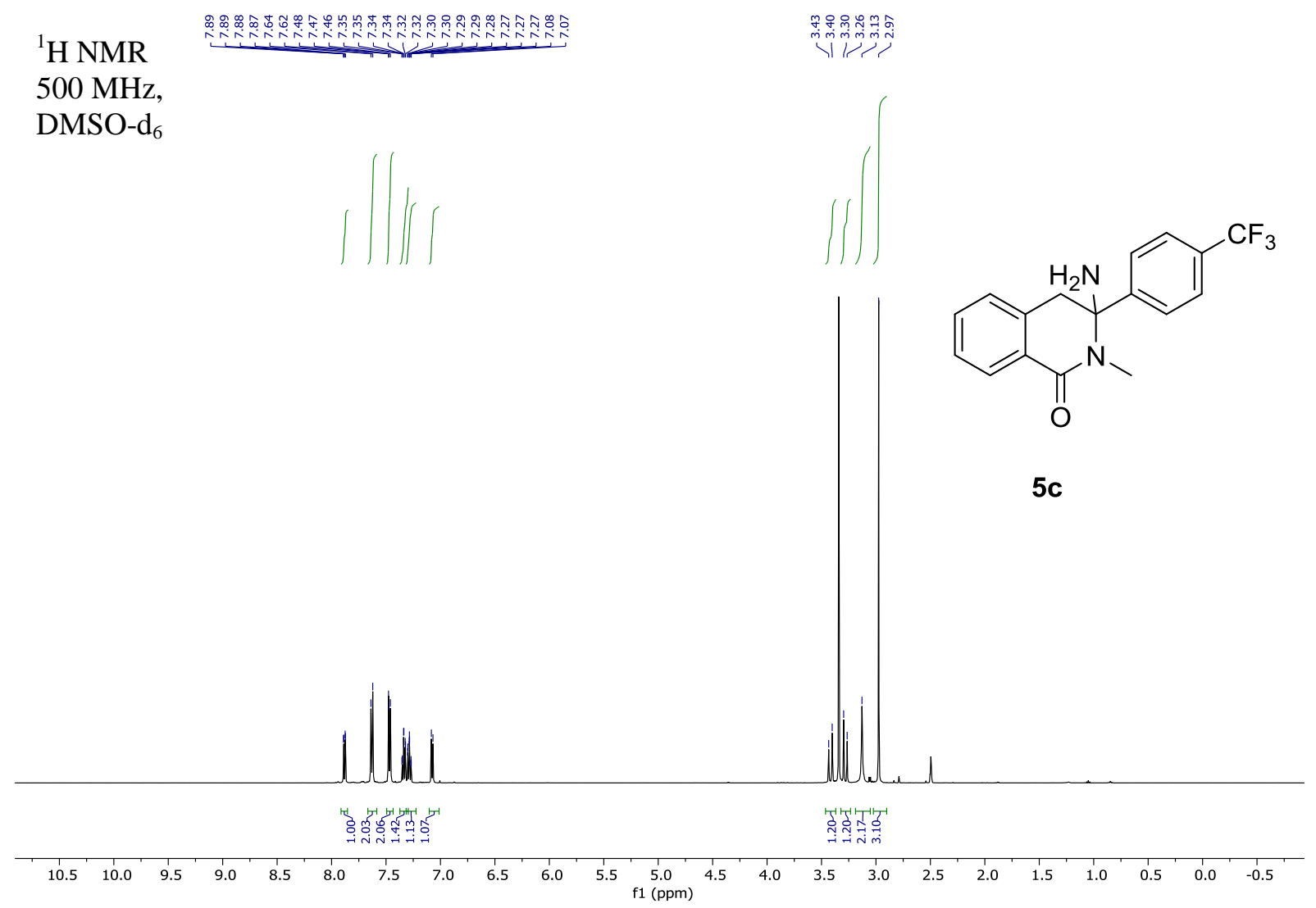

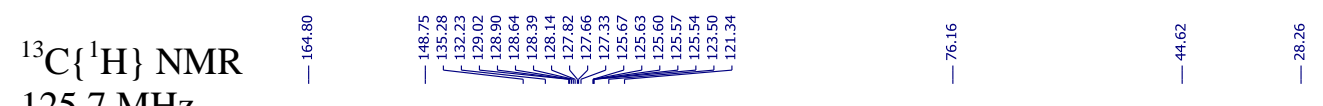
$125.7 \mathrm{MHz}$,

DMSO-d $_{6}$

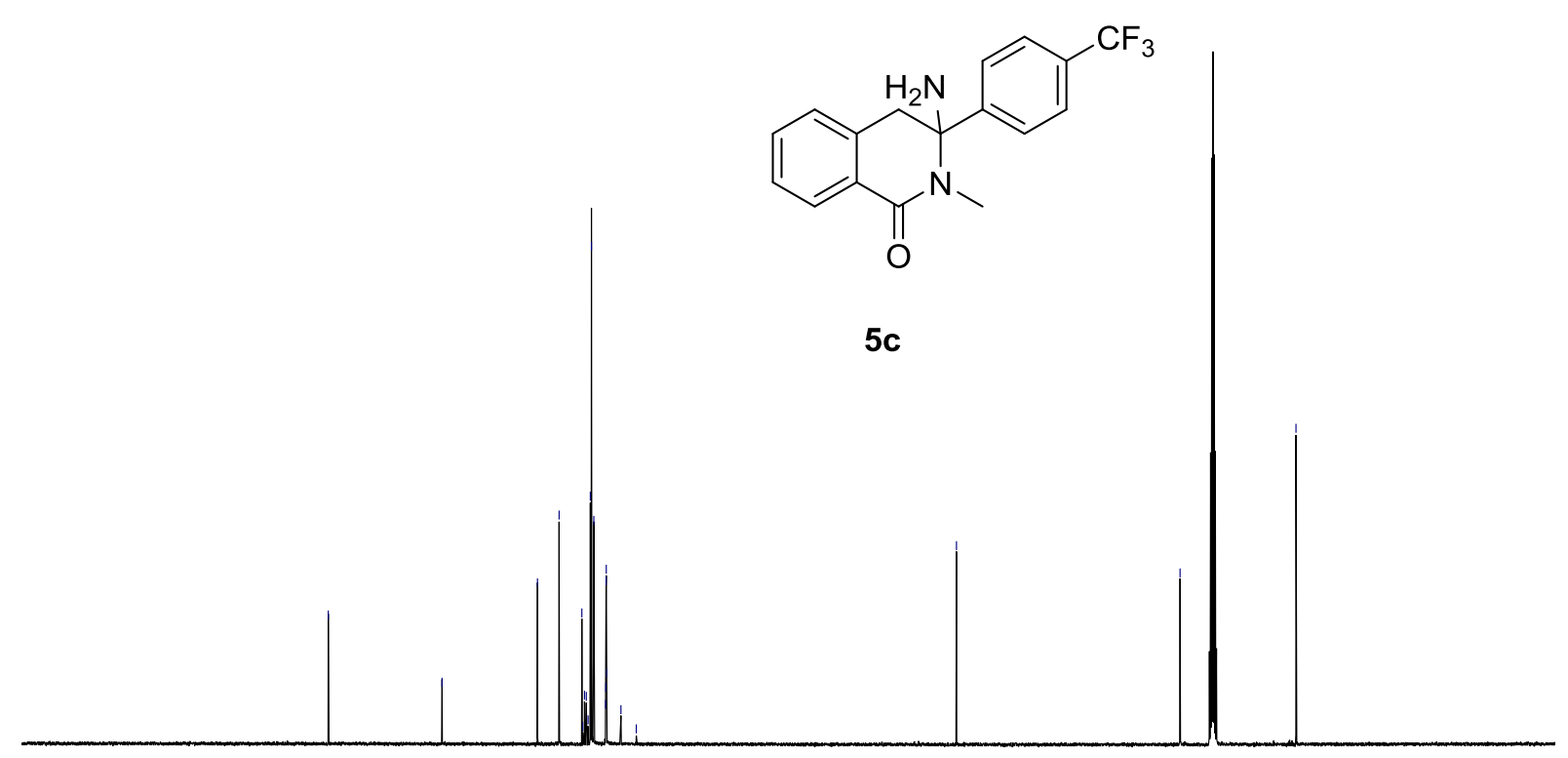

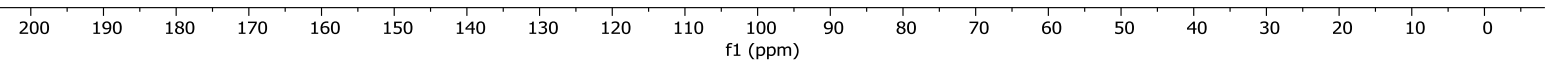




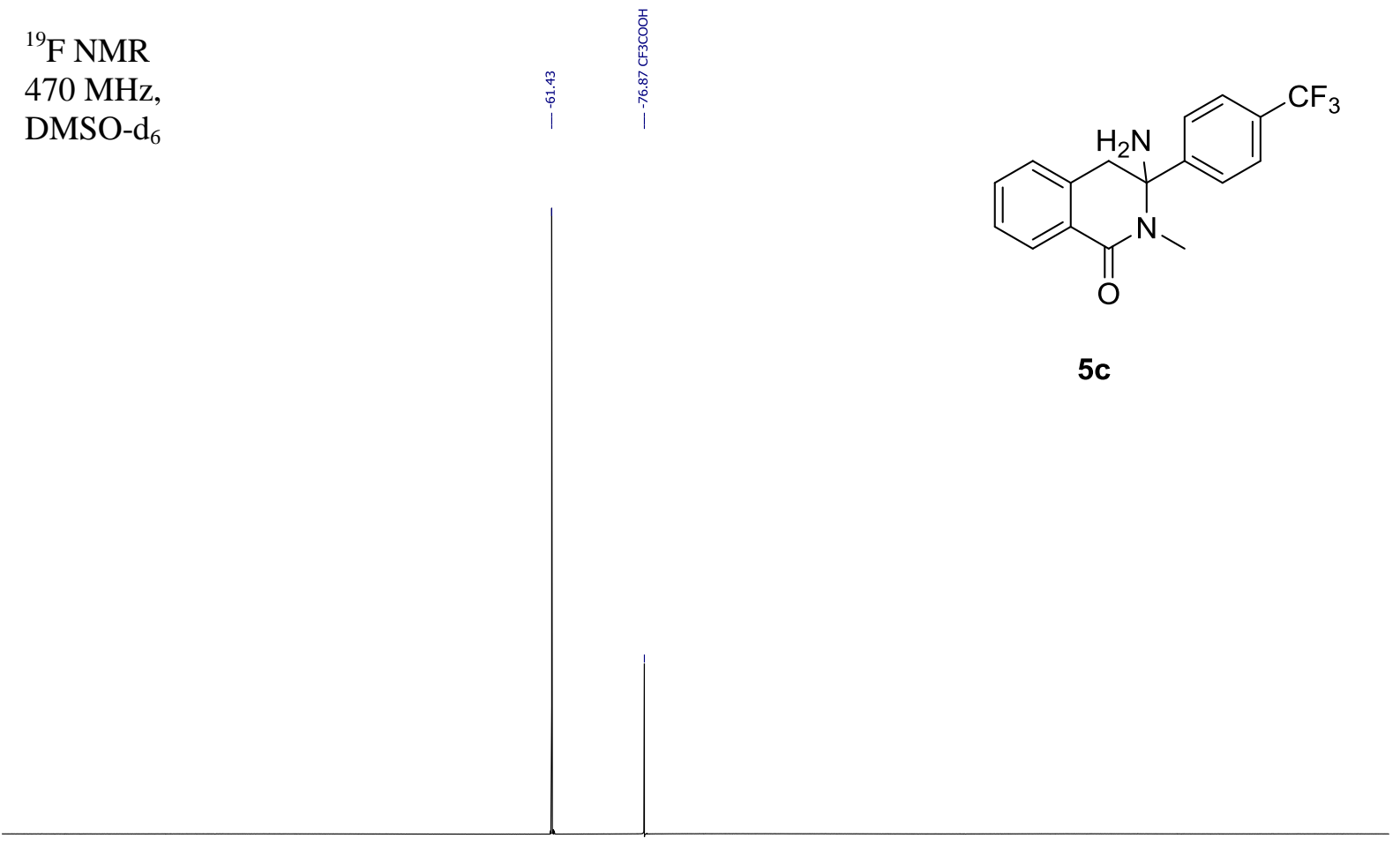

\begin{tabular}{lllllllllllllllllllllllllll}
\hline 30 & 20 & 10 & 0 & -10 & -20 & -30 & -40 & -50 & -60 & -70 & -80 & -90 & -100 & -110 & -120 & -130 & -140 & -150 & -160 & -170 & -180 & -190 & $-2(1$ \\
\hline
\end{tabular} 


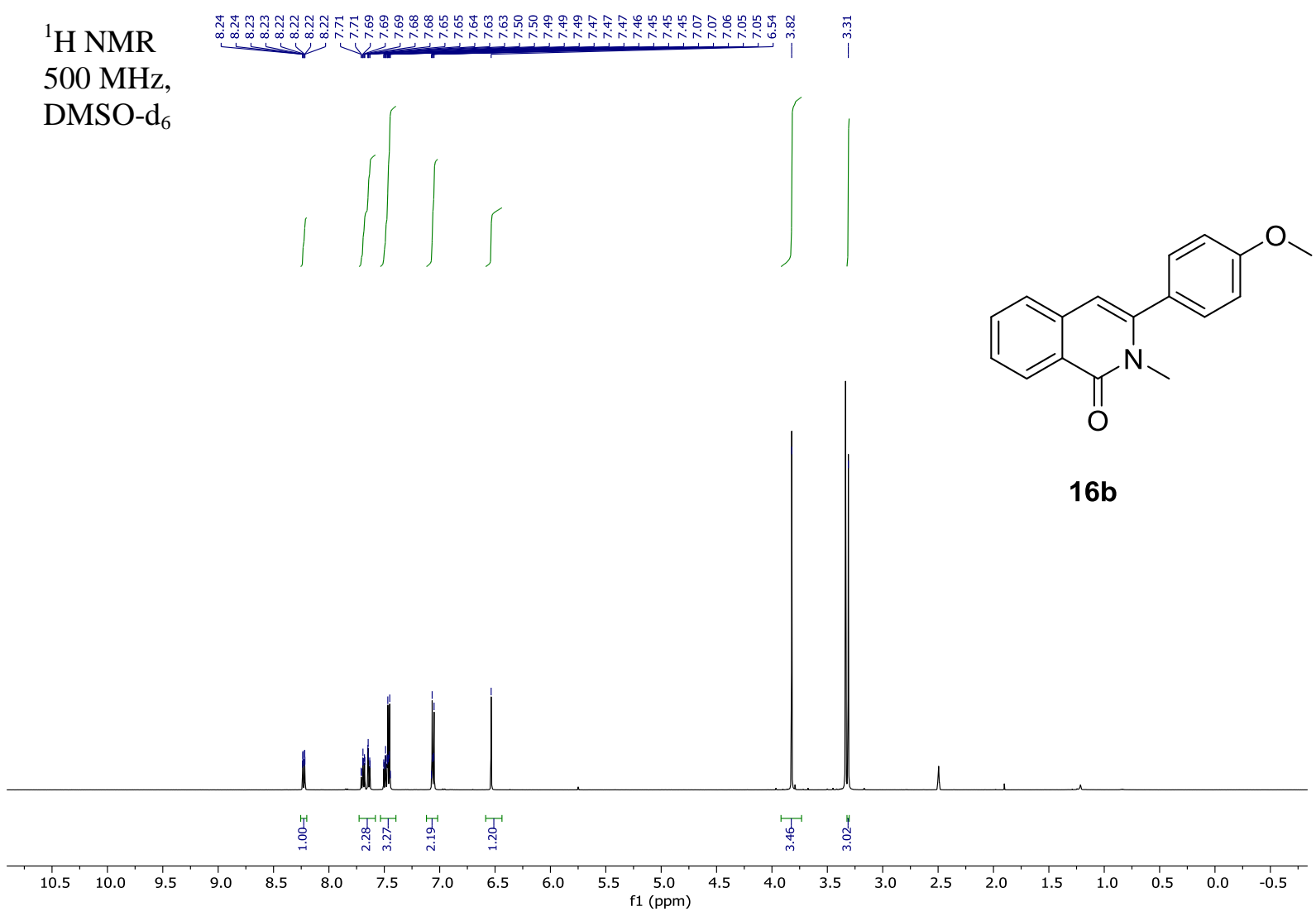

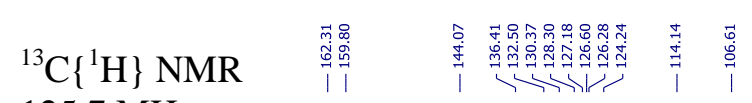
$125.7 \mathrm{MHz}$,

DMSO-d $_{6}$<smiles>COc1ccc(-c2cc3ccccc3c(=O)n2C)cc1</smiles>

16b

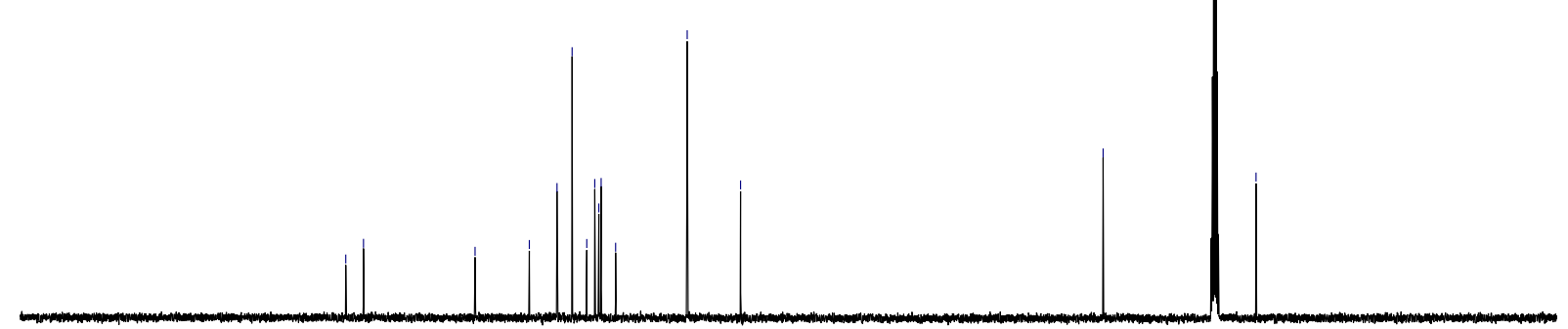

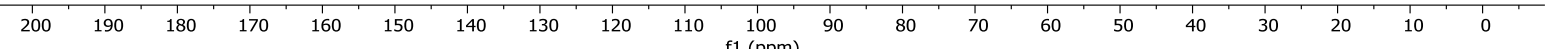




\section{Crystallography}

Full-sets of diffraction data for 5a were collected at 150(2)K with a Bruker D8-Venture diffractometer equipped with $\mathrm{Mo}\left(\mathrm{Mo} / \mathrm{K}_{\alpha}\right.$ radiation; $\left.\lambda=0.71073 \AA\right)$ microfocus X-ray $(\mathrm{I} \mu \mathrm{S})$ sources, Photon CMOS detector and Oxford Cryosystems cooling device was used for data collection.

The frames were integrated with the Bruker SAINT software package using a narrowframe algorithm. Data were corrected for absorption effects using the Multi-Scan method (SADABS). Obtained data were treated by XT-version 2014/5 and SHELXL-2017/1 software implemented in APEX3 v2016.5-0 (Bruker AXS) system. ${ }^{\text {[S1] }}$

Hydrogen atoms were mostly localized on a difference Fourier map, however to ensure uniformity of treatment of crystal, all hydrogen were recalculated into idealized positions (riding model) and assigned temperature factors $\mathrm{H}_{\mathrm{iso}}(\mathrm{H})=1.2 \mathrm{U}_{\text {eq }}$ (pivot atom) or of $1.5 \mathrm{U}_{\text {eq }}$ (methyl). $\mathrm{H}$ atoms in methyl, methylene, moieties and hydrogen atoms in aromatic rings were placed with $\mathrm{C}-\mathrm{H}$ distances of $0.96,0.97$ and $0.93 \AA$, hydrogen atoms in N-H bonds were refined freely.

$R_{\text {int }}=\sum\left|F_{\mathrm{o}}{ }^{2}-F_{\text {o,mean }}{ }^{2}\right| / \sum F_{\mathrm{o}}{ }^{2}, \mathrm{GOF}=\left[\sum\left(w\left(F_{\mathrm{o}}{ }^{2}-F_{\mathrm{c}}{ }^{2}\right)^{2}\right) /\left(N_{\text {diffrs }}-N_{\text {params }}\right)\right]^{1 / 2}$ for all data, $R(F)=$ $\sum|| F_{\mathrm{o}}|-| F_{\mathrm{c}}|| / \sum\left|F_{\mathrm{o}}\right|$ for observed data, $w R\left(F^{2}\right)=\left[\sum\left(w\left(F_{\mathrm{o}}{ }^{2}-F_{\mathrm{c}}{ }^{2}\right)^{2}\right) /\left(\sum w\left(F_{\mathrm{o}}{ }^{2}\right)^{2}\right)\right]^{1 / 2}$ for all data.

Crystallographic data for structural analysis have been deposited with the Cambridge Crystallographic Data Centre, CCDC no. 2065025. Copies of this information may be obtained free of charge from The Director, CCDC, 12 Union Road, Cambridge CB2 1EY, UK (fax: +44-1223-336033; e-mail: deposit@ccdc.cam.ac.uk or ww: http://www.ccdc.cam.ac.uk).

The crystals were prepared by crystallization of 5a from ethyl acetate by leaving a hot solution to stand at rt. 
Table SI-1. Experimental details for 5 a.

\begin{tabular}{|c|c|}
\hline \multicolumn{2}{|l|}{ Crystal data } \\
\hline Chemical formula & $\mathrm{C}_{16} \mathrm{H}_{16} \mathrm{~N}_{2} \mathrm{O}$ \\
\hline$M_{\mathrm{r}}$ & 252.31 \\
\hline Crystal system, space group & Triclinic, $P 1$ \\
\hline Temperature (K) & 150 \\
\hline$a, b, c(\AA)$ & $8.3608(3), 8.8135(3), 9.6041$ (3) \\
\hline$\alpha, \beta, \gamma\left({ }^{\circ}\right)$ & $109.140(1), 94.023(1), 107.043(1)$ \\
\hline$V\left(\AA^{3}\right)$ & $628.30(4)$ \\
\hline$Z$ & 2 \\
\hline Radiation type & Mo $K \alpha$ \\
\hline$\mu\left(\mathrm{mm}^{-1}\right)$ & 0.09 \\
\hline Crystal size (mm) & $0.68 \times 0.41 \times 0.14$ \\
\hline \multicolumn{2}{|l|}{ Data collection } \\
\hline Diffractometer & Bruker D8 - Venture \\
\hline Absorption correction & Multi-scan \\
\hline$T_{\min }, T_{\max }$ & $0.690,0.746$ \\
\hline $\begin{array}{l}\text { No. of measured, independent and observed }[I> \\
2 \sigma(I)] \text { reflections }\end{array}$ & $21557,3127,2681$ \\
\hline$R_{\text {int }}$ & 0.037 \\
\hline$(\sin \theta / \lambda)_{\max }\left(\AA^{-1}\right)$ & 0.668 \\
\hline \multicolumn{2}{|l|}{ Refinement } \\
\hline$R\left[F^{2}>2 \sigma\left(F^{2}\right)\right], w R\left(F^{2}\right), S$ & $0.039,0.107,1.04$ \\
\hline No. of reflections & 3127 \\
\hline No. of parameters & 181 \\
\hline $\mathrm{H}$-atom treatment & $\begin{array}{l}\mathrm{H} \text { atoms treated by a mixture of independent and } \\
\text { constrained refinement }\end{array}$ \\
\hline$\Delta \rho_{\max }, \Delta \rho_{\min }\left(\mathrm{e} \AA^{-3}\right)$ & $0.32,-0.23$ \\
\hline
\end{tabular}


Although more than four hundreds 3,4-dihydroisoquinolin-1(2H)-ones crystal structures are reported within the Cambridge Structural Database, only four of them resemble the structure of 5a with an amino substitution at position $3 .{ }^{[\mathbf{S} 2]}$ The structure of $\mathbf{5 a}$, unambiguously determined by sc-XRD techniques, crystallize in triclinic system within centrosymmetric space group $P-1$ (Fig. SI-1). The interatomic distances very close to distances in analogous compounds mentioned above and comparable to appropriate separations found for organic compounds, ${ }^{[\mathbf{S} 3]}$ except of C2-N1 (Fig. SI-1 caption), which is significantly elongated due the ring tension. Surprisingly enough, the $\mathrm{N}-\mathrm{H}$ and amido groups does not have the expected $\mathrm{H}$-bridging contact, instead of which only the $\mathrm{C}=\mathrm{O} \ldots \mathrm{H}-\mathrm{C}$ weak interactions are the structure making for 5a supramolecular architecture.

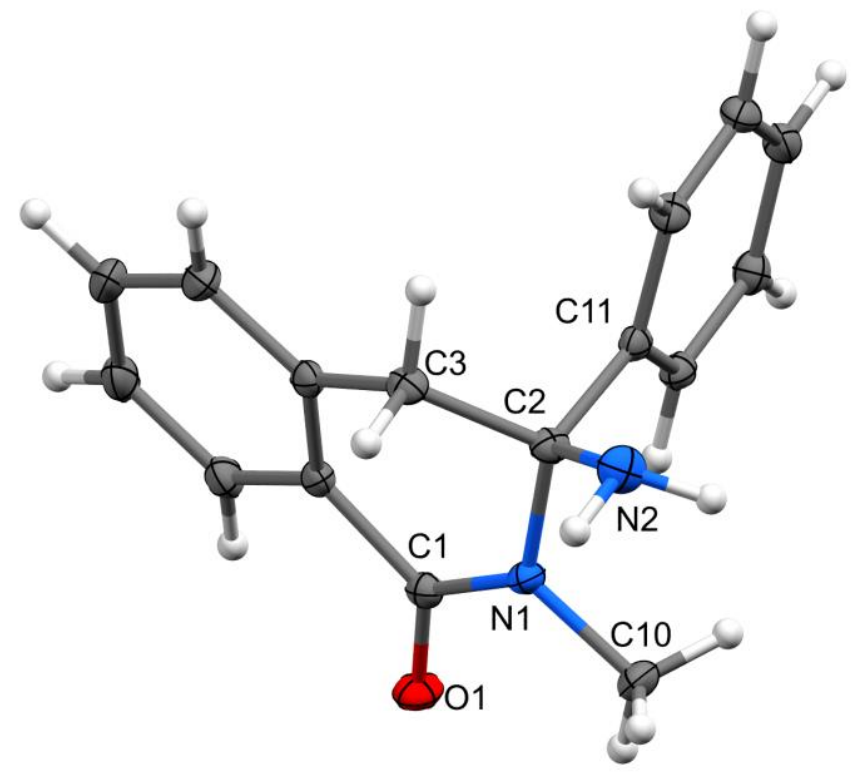

Figure SI-1. ORTEP drawing for 5a. Thermal ellipsoids are drawn at the $40 \%$ probability level. Selected interatomic distances $[\AA]$ : O1-C1 1.2365(13), N1-C1 1.3594(13), N1-C10 1.4608(14), N1-C2 1.5012(13), C1-C5 1.4884 (14), C2-N2 1.4504 (13), C2-C3 1.5307(15), C2-C11 1.5352(14), C3-C4 1.4935(15).

\section{References}

[S1] Sheldrick, G.M. SHELXT - Integrated space-group and crystal-structure determination. Acta Cryst. A71, 2015, 3.

[S2] (a) Sun, R.; Yang, X.; Chen, X.; Zhang, C.; Zhao, X.; Wang, X.; Zheng, X.; Yuan, M.; Fu, H.; Li, R.; Chen H. Rh(III)-Catalyzed $[4+2]$ Self-Annulation of $N$ Vinylarylamides. Org. Lett. 2018, 20, 6755. (b) Rimböck, K.-H.; Pöthig, A.; Bach T. 
Photocycloaddition and Rearrangement Reactions in a Putative Route to the Skeleton of Plicamine-Type Alkaloids. Synthesis 2015, 47, 4869. (c) Daniels, A. M.; Supinski, M. A.; Kennedy, D. P.; Robinson, W. D.; Valente, E. J. Hydroxylamine and Pseudoacyl Systems: Pseudo-oximes. J. Chem. Cryst. 2013, 43, 6.

[S3] Allen, F. H.; Watson, D. G.; Brammer, L.; Orpen, A. G.; Taylor, R. Typical interatomic distances: organic compounds In International Tables for Crystallography 2006. Vol. C, ch. 9.5 , pp. $790-811$. 